\title{
Continuous Aerobic Training and High Intensity Interval Training Increase Exercise Tolerance in Heart Failure Patients: A Retrospective Study
}

\author{
Diego Busin, ${ }^{1,2}$ Alexandre M. Lehnen, ${ }^{3 \oplus}$ Olga S. Tairova, ${ }^{2}$ Eduardo P. Comparsi, ${ }^{\circledR}$ Daniela Carneiro, ${ }^{2}$ Micael \\ Potter, ${ }^{2 \oplus}$ Luís F. Deresz, $^{4}$ Pedro Dal Lago, ${ }^{1 \oplus}$ Ramiro B. Nunes ${ }^{1}{ }^{\circledR}$ \\ Universidade Federal de Ciências da Saúde de Porto Alegre,' Porto Alegre, RS - Brazil. \\ Universidade de Caxias do Sul, Caxias do Sul, ${ }^{2}$ RS - Brazil. \\ Instituto de Cardiologia do Rio Grande do Sul/Fundação Universitária de Cardiologia, ${ }^{3}$ Porto Alegre, RS - Brazil. \\ Universidade Federal de Juiz de Fora, ${ }^{4}$ Governador Valadares, MG - Brazil.
}

\section{Abstract}

Background: Conventional aerobic training is the first choice in cardiac rehabilitation for patients with chronic heart failure (CHF). However, high-intensity interval training (HIIT) may be an alternative, although it has little evidence.

Objectives: To evaluate the effect of continuous aerobic training (CAT) or HIIT on exercise tolerance in CHF patients.

Methods: Retrospective study with 30 patients, of both genders, members of a 10-week CAT or HIIT program. The control group $(\mathrm{CON})$ consisted of patients who did not participate voluntarily in the program. Peak oxygen uptake (VO2peak), thresholds (LV1 and LV2), and ventilatory efficiency in the production of dioxide (VE/VCO2 slope), oxygen uptake efficiency (OUES), and VO2 recovery kinetics were analyzed. A two-way or repeated measures ANOVA was used, followed by Fisher's post-hoc test $(\mathrm{p}<0.05)$.

Results: After 10 weeks of training, the CAT group increased the treadmill speed at LV1 ( $p=0.040$ ), while the HIIT increased both the speed $(\mathrm{p}=0.030)$ and incline of the treadmill $(\mathrm{p}<0.001)$ for VO2peak and LV2, as well as the total time of the cardiopulmonary test. The $\mathrm{VE} / \mathrm{VCO}_{2}$ slope was lower than that predicted for CAT ( $\left.\mathrm{p}=0.003\right)$ and HIIT ( $\left.\mathrm{p}=0.008\right)$. There was no change in $\mathrm{VO}_{2}$ peak, recovery of heart rate (HR), and $\mathrm{VO}_{2}, \mathrm{VE} / \mathrm{VCO}_{2}$, and OUES in both groups.

Conclusions: After 10 weeks, both CAT and HIIT increased the tolerance to physical exercise. However, HIIT showed improvement in more parameters, differently from CAT. (Int J Cardiovasc Sci. 2021; [online].ahead print, PP.0-0)

Keywords: Heart Failure; Exercise. Physical Exertion; Exercise Therapy; Rehabilitation; Exercise Movement Techniques.

Introduction

Chronic heart failure (CHF) is a multifactorial syndrome characterized by cardiovascular and ventilatory abnormalities associated with the increase in the neurohumoral systems. ${ }^{1,2}$ The cycle generated between physical decoding and disorders is associated with classic CHF physiopathological mechanisms. Nevertheless, this condition is pinpointed as the main problem of the reduction in tolerance to physical exercise in this population. ${ }^{1,2}$

International guidelines have voiced concern over the theme and have highlighted the heart rehabilitation programs with emphasis on regular physical exercise, and under professional supervision, as being forms of safe, non-pharmacological treatment in adjunct therapy for $\mathrm{CHF}$ patients. ${ }^{3}$ The benefits of physical exercise are demonstrated in studies that observe improvements in the variable relation to exercise tolerance, functional capacity, and ventilatory efficiency of these patients. ${ }^{4-6}$ Improvement in one's cardiorespiratory fitness, illustrated by increases in the $\mathrm{VO}_{2}$ peak and in the total duration of the cardiopulmonary exercise test, present strong prognostic values in $\mathrm{CHF}$, thus confirming the benefits and safety of physical exercise.

\section{Mailing Address: Alexandre Machado Lehnen}

Instituto de Cardiologia do Rio Grande do Sul - Unidade de Pesquisa

Av. Princesa Isabel, 395. Postal Code: 90620 001, Santana, Porto Alegre, RS - Brazil.

E-mail: amlehnen@gmail.com 
Mild-intensity continuous aerobic training (CAT), maintaining an intensity between $60 \%$ and $80 \%\left(\mathrm{VO}_{2}\right.$ peak or reserve heart rate), ${ }^{7}$ has been described as the most successful style of training, due to its efficacy and safety. ${ }^{7-9}$ Nonetheless, studies have used greater intensities of physical exercise in CHF patients with promising results and, in some cases, even greater than the CAT. ${ }^{10-12}$ In this context, interest concerning increasing intensity (75 to $90 \% \mathrm{VO}_{2}$ peak) of physical exercise is growing, especially as regards safe training for this population ${ }^{12}$. Some justifications for this type of conduct would be a greater aerobic and cardiovascular adaptation that could be achieved by using this type of strategy, ${ }^{10}$ in addition to improvements in exercise capacity, endothelial function, left ventricular function, and ejection fraction. ${ }^{12}$ These conclusions, however, seem rather uncertain, since the impacts upon the difference in prognoses, the length of training, and the diversity of protocols, with different durations, recoveries, and intensities used in the studies, make the defense of clinical training standards for $\mathrm{CHF}$ patients difficult; thus, a larger body of evidence is needed.

Faced with the continuing key question about which aerobic training method should be ideally prescribed in heart rehabilitation programs, the search for this type of evidence is necessary. Hence, this study sought to evaluate the potential benefits of mild-intensity CAT and high-intensity interval training (HIIT), through the medical records of Class II and III (NYHA) CHF patients who participated in a heart rehabilitation program.

\section{Methods}

This study was conducted in accordance with that set forth in the Helsinki Declaration and CNS 466/12. All patients read and signed the Free and Informed Consent Form provided by the Clinical Center where the data were collected upon entrance into the program. The project was approved by the Research Ethics Committee from Universidade Federal de Ciências da Saúde de Porto Alegre (logged under protocol number 3.055.126).

Through a retrospective cohort, this study assessed the records of data referent to the physical training sessions of patients with CHF and who agreed to participate in the heart rehabilitation program of the Sports Medicine Unit of the Clinical Center of Universidade de Caxias do Sul, RS, Brazil, between 2016 and 2017.

\section{Samples and Groups}

This study included medical records from $30 \mathrm{CHF}$ patients (64 \pm 9 years of age), from both genders, left ventric (CON) consisted of patients who opted not to participate in the heart rehabilitation program at that moment.

The exclusion criteria were: incomplete medical records and patients who did not present reference values during the strength test $\left(\mathrm{R}<1.0\right.$ to reach the $\mathrm{VO}_{2}$ peak or submaximal heart rate).

\section{Heart Rehabilitation Program}

Once the patients had been admitted to the program, a clinical anamnesis was performed by the doctor of the program. After, the patients were referred for initial physical assessments and were distributed randomly (https://www.randomizer.org/) for supervised training in the different methods: CAT $(\mathrm{n}=10)$ and HIIT $(\mathrm{n}=10)$. Patients that chose not the participate in the rehabilitation program were allocated to the control group $(\mathrm{CON}, \mathrm{n}=10)$.

\section{Cardiopulmonary Exercise Test}

After warming up for 10 minutes, the $\mathrm{VO}_{2}$ peak was evaluated by the gas analyzer (VO2000, Medical Graphics Diagnostics Corporation, USA), using a Super ATL 300 treadmill (Imbramed, Porto Alegre, RS, Brazil). A Ramp protocol was used, beginning at $3 \mathrm{~km} / \mathrm{h}$ and with no slope. The speed was then increased by $0.3 \mathrm{~km} / \mathrm{h}$, and the slope by $1.6 \%$, for each minute of exercise, until patient exhaustion. The exhaled gases were quantified every 20 seconds, obtaining ventilator flows and volumes. The patient's blood pressure (BP), heart rate (HR), minute ventilation $(\mathrm{VE})$, oxygen uptake $\left(\mathrm{VO}_{2}\right)$, carbon dioxide production $\left(\mathrm{VCO}_{2}\right)$, and respiratory exchange ratio $(\mathrm{R})$, as well as the ventilator equivalents for oxygen $\left(\mathrm{VE} / \mathrm{VO}_{2}\right)$ and carbon dioxide $\left(\mathrm{VE} / \mathrm{VCO}_{2}\right)$, were analyzed during the evaluation. Based on this data, the $\mathrm{VO}_{2}$ peak, the first ventilatory threshold (LV1), the second ventilatory threshold (LV2), among other ventilatory and metabolic measures described in the literature, were determined..$^{13}$

\section{Evaluation of Ventilatory Efficiency}

The ventilatory equivalent for $\mathrm{CO}_{2}$ was calculated by means of the ratio between the instantaneous ventilation rate (VE) and the release of $\mathrm{CO}_{2}(\mathrm{VE} / \mathrm{VCO} 2)$. For this, the present study used the data recorded in the cardiopulmonary exercise test, from the beginning to the LV2. The slope (VE/ $/ \mathrm{VCO}_{2}$ slope) was used to analyze the ventilatory efficiency. ${ }^{14}$ The predicted values for this variable were obtained by means of the equation described in the literature. ${ }^{15}$ 


\section{Evaluation of Oxygen Uptake Efficiency}

The oxygen uptake efficiency slope (OUES) is represented by the rate of increase of $\mathrm{VO}_{2}$ in response to $\mathrm{VE}$, and was calculated by measuring the relationship between $\mathrm{VO}_{2}$ and the VE logarithm. ${ }^{16}$ The predicted value for this variable has already been described in the literature. ${ }^{16}$

\section{Recovery Kinetics of Oxygen Uptake and Heart Rate}

At the end of the cardiopulmonary exercise test, the patients were instructed to remain seated for three minutes while the metabolic and ventilatory variables continued to be recorded. The data during this period served to analyze and determine the recovery kinetics of $\mathrm{VO}_{2^{\prime}}$ in the following manner: the magnitude of the recovery through the relationship of $\mathrm{VO}_{2}$ regarding time $\left(\mathrm{VO}_{2} / \mathrm{t}\right.$ slope $)$, calculated by the linear regression model and adjusted to a simple exponential curve. ${ }^{17}$ The slope equation was calculated considering the time needed to reach the $\mathrm{VO}_{2}$ peak and recovery in the first three minutes; the established value of the $\mathrm{VO}_{2}$ recovery was obtained by determining the difference between the value of the $\mathrm{VO}_{2}$ peak and that of $\mathrm{VO}_{2}$ in the first, second, and third minutes after the end of the $\mathrm{VO}_{2}$ peak test. ${ }^{18} \mathrm{In}$ addition, the recovery kinetics for HR were calculated by subtracting the HR value at the end of the test from the values of minutes 1,2 , and 3 in the recovery test. ${ }^{14}$

\section{Protocols of the Heart Rehabilitation Training Program}

The CAT consisted of a mild-intensity continuous training (70-75\% HRreserve) for 47 minutes per session. The time was calculated and duly controlled to guarantee that the training protocols were isocaloric. ${ }^{12}$ A fingertip pulse oximeter (NoninOnix 9500, United Kingdom) was used to monitor the patients' $\mathrm{HR}$ and peripheral saturation of oxygen $\left(\mathrm{SpO}_{2}\right)$. The speed was adjusted continuously to guarantee that each session would be performed in the HR target.

The HIIT consisted of 38 minutes, beginning with the 10-minute warm-up, between 50-60\% HRreserve, followed by 4 series of 4 minutes between $90-95 \%$ HRreserve. Each series was staggered, with an active pause of 3 minutes (light walk). The training session was finished with a period of 3 minutes of cool down (50\% HRreserve).

\section{Data Collection}

This study was conducted by consulting the data recorded in the medical records of each patient who trained three times per week, during 10 consecutive weeks. These medical charts were used in the routine of the heart rehabilitation program. After the previous study of the feasibility of the medical records, the data were tabulated within a databank put together in Excel 2013 (Microsoft Office).

\section{Statistical Analysis}

The normality of the data was evaluated using the Shapiro-Wilk test. The data were expression as average \pm standard deviation. The two-way ANOVA was used, together with Fisher's post-hoc test. By contrast, the comparison of the data of the $\mathrm{VO}_{2}$ recovery kinetics and HR between the 1,2, and 3 minute intra-groups was performed using the repeated measures ANOVA, together with Fisher's post-hoc test. The GraphPad Prism 6 program (GraphPad Software, San Diego, California, USA) was used as a computational tool for data analysis, considered significant for a $\alpha=0.05$.

\section{Results}

This study analyzed the medical records of 30 patients with $\mathrm{CHF}$, with $\mathrm{LVEF}<40 \%$, who voluntarily chose to participate in the heart rehabilitation program between August 2016 and December 2017. One patient was over 80 years of age, nine were between 70 and 80 years of age, eleven were from 60 to 70 years of age, and nine were between 50 and 60 years of age. Of these, five patients were using a pacemaker. All of the patients were hemodynamically stable, with optimized treatment, including $\beta$-blockers and antiplatelet aggregation over the past 12 months, even during the period of physical training. No event related to the sessions of physical training occurred in the patients in either group. The presence of participants reached $100 \%$ of the scheduled sessions (10 weeks).

Figure 1 presents parameters of intensity (speed and slope) and time to reach the $\mathrm{VO}_{2}$ peak referent to the cardiopulmonary test. After ten weeks of physical training, the CAT group presented improvement in exercise tolerance for LV1 when compared to the initial test. By contrast, the HIIT group presented improvement in the tolerance to the exercise, represented by the higher speed and slope for LV2, as well as in the time to reach the $\mathrm{VO}_{2}$ peak, when compared to the beginning of the physical training. It is possible to observe in Figure 1 that the HIIT group presented a set of greater benefits when compared to the CAT. 


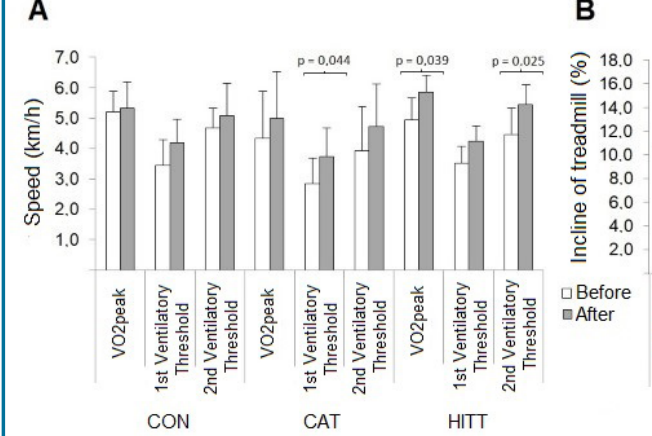

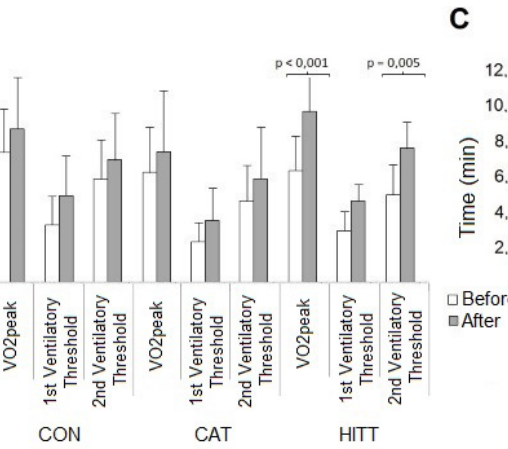

$\mathrm{CON}$

CAT

HITT

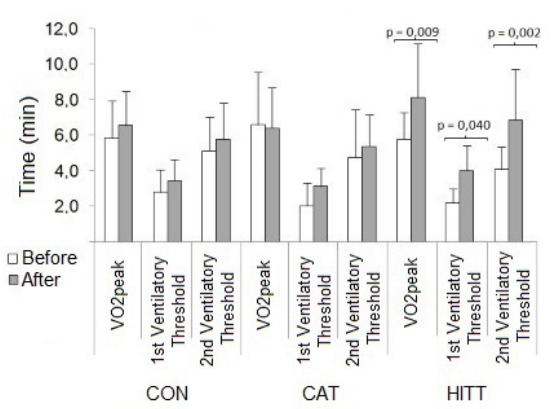

Figure 1 - Cardiovascular exercise test.

Speed (Panel A) and incline of treadmill (Panel B), as well as the time to reach the VO2peak, 1st ventilatory threadshold, and 2 nd ventilatory threadshlod, before and after 10 weeks of physical training. CON: control group; CAT: moderate-intensity continunous aerobic training; HIIT: highintensity interval training. The differences were tested by ANOVA followed by Fisher's post-hoc test $(0<0.050)$

Details of the cardiopulmonary test are presented in Table 1. No significant difference was observed in the records of the recovery kinetics of $\mathrm{HR}$ and $\mathrm{VO}_{2}$. The patient presented no difference in the parameters of body composition and physical fitness.

\section{Discussion}

The main finding in this study was that Class II and III (NYHA) CHF patients, who took part in a 10-week program of CAT or HIIT, increased their tolerance to physical exercise, when compared to the pre-training moment. Moreover, the HIIT improved a greater number of evaluated parameters when compared to the CAT, illustrating its superiority to conventional training, thus confirming our hypothesis. The parameters of the cardiopulmonary exercise test proved to be useful in the long-term diagnosis and prognosis of $\mathrm{CHF}$ and in the intolerance to physical exercise. The $\mathrm{VO}_{2}$ peak has been used for decades as a universal Market, capable of reflecting the severity of the disease, ${ }^{17}$ and has been considered the "gold-standard" measurement to identify patients with a worse prognosis, ${ }^{19}$ in addition to being a parameter for the prescription of physical training involving this population..$^{20,21}$ In this sense, our study's results present a clinical importance, as they present an improvement in the $\mathrm{VO}_{2}$ peak, which is greater than $1 \mathrm{ml} . \mathrm{kg}^{-1} \cdot \mathrm{min}^{-1}$, considered relevant for autonomy and performance of many daily life activities (DLAs).$^{22}$ Likewise, the $\mathrm{VO}_{2}$ peak data observed in our study are slightly greater than the $0.6 \mathrm{ml} . \mathrm{kg}^{-1} \cdot \mathrm{min}^{-1}$ found in the HF-ACTION trial ${ }^{8}$ and corroborate with the meta-analysis findings from Cornelis. ${ }^{23}$

The present study demonstrates a wide range of parameters of gas exchanges verified in the submaximal cardiopulmonary exercise test. Woods et al., ${ }^{24}$ found strong predictors of adverse heart outcomes and survival in $\mathrm{CHF}$ patients through submaximal parameters of gas exchange, especially the $\mathrm{VE} / \mathrm{VCO}_{2}, \mathrm{VE} / \mathrm{VCO}_{2}$ slope, and OUES, found with "R=0.9", formulating prognoses similar to the $\mathrm{VO}_{2}$ peak. ${ }^{24}$ Other authors have suggested that changes in these prognostic markers for CHF seem to be more important predictors of the capacity of exercise tolerance than the results from the $\mathrm{VO}_{2}$ peak. ${ }^{25}$

As a result, ventilatory efficiency for the elimination of $\mathrm{CO}_{2}\left(\mathrm{VE} / \mathrm{VCO}_{2}\right.$ or $\mathrm{VE} / \mathrm{VCO}_{2}$ slope $)$ in response to submaximal exercise was investigated to determine the prognostic values for CHF patients. ${ }^{25}$ However, this study found values of lower than 34 and 45, indicating a prognosis of a $50 \%$ probability of mortality in two years, regardless of the $\mathrm{VO}_{2}$ peak result. In a study from Chua et al., ${ }^{26}$ it was verified that $\mathrm{CHF}$ patients, evaluated by the cardiopulmonary exercise test, with a $\mathrm{VE} / \mathrm{VCO}_{2}$ slope $>34$, presented a greater risk of hospitalization caused by hemodynamic decompensation, as well as death. Ferreira et al., ${ }^{27}$ found the cut-off point for the $\mathrm{VE} / \mathrm{VCO}_{2}$ slope $\geq 43$ to be the ideal to determine the recommendation for a heart transplant. The time to obtain the lowest value of $\mathrm{VE} / \mathrm{VCO}_{2}$ (>5:45 minutes) also represents a poor prognosis for this population. ${ }^{28}$ In this indicator, our 
Table 1 - Responses from the cardiopulmonary test before and after 10 weeks of physical training

\begin{tabular}{|c|c|c|c|c|c|c|}
\hline & \multicolumn{2}{|c|}{$\mathrm{CON}$} & \multicolumn{2}{|c|}{ CAT } & \multicolumn{2}{|c|}{ HIIT } \\
\hline & Before & After & Before & After & Before & After \\
\hline $\mathrm{VO}_{2}$ peak $\left(\mathrm{ml} \cdot \mathrm{kg}^{-1} \mathrm{~min}^{-1}\right)$ & $19.3 \pm 4,5$ & $19.5 \pm 4,8$ & $16.2 \pm 5,9$ & $17.3 \pm 5,9$ & $18.6 \pm 2,7$ & $19.8 \pm 3,2$ \\
\hline Maximal HR (bpm) & $121 \pm 18$ & $127 \pm 24$ & $116 \pm 31$ & $113 \pm 24$ & $119 \pm 11$ & $129 \pm 16$ \\
\hline Maximal VE/ $/ \mathrm{VCO}_{2}$ & $29.6 \pm 5,2$ & $29.1 \pm 5,3$ & $28.4 \pm 5,4$ & $28.6 \pm 5,2$ & $25.4 \pm 2,7$ & $25.7 \pm 3,7$ \\
\hline Minimum $\mathrm{VE} / \mathrm{VCO}_{2}$ & $27.5 \pm 4,6$ & $26.7 \pm 4,1$ & $26.7 \pm 4,9$ & $27.3 \pm 5,3$ & $23.4 \pm 3,0$ & $23.6 \pm 3,8$ \\
\hline Time to reach $\mathrm{VE} / \mathrm{VCO}_{2}(\mathrm{~min})$ & 04:04 $\pm 01: 36$ & $03: 53 \pm 02: 03$ & $02: 57 \pm 01: 37$ & $03: 47 \pm 01: 37$ & 03:34 $\pm 01: 07$ & $05: 22 \pm 02: 56$ \\
\hline $\mathrm{VE} / \mathrm{VCO}_{2}$ slope & $30.1 \pm 10,0$ & $30.5 \pm 16,0$ & $26.6 \pm 9,0$ & $23.1 \pm 4,5$ & $22.0 \pm 3,2$ & $21.7 \pm 5,3$ \\
\hline $\begin{array}{l}\mathrm{VE} / \mathrm{VCO}_{2} \text { slope } \\
\% \text { of prediction }\end{array}$ & $113 \pm 33$ & $110 \pm 52$ & $95 \pm 34$ & $82 \pm 19^{*}$ & $98 \pm 15$ & $79 \pm 24^{*}$ \\
\hline OUES & $2148 \pm 1192$ & $1887 \pm 841$ & $1559 \pm 536$ & $1800 \pm 390$ & $2464 \pm 807$ & $1926 \pm 700$ \\
\hline $\begin{array}{l}\text { OUES } \\
\% \text { of prediction }\end{array}$ & $107 \pm 105$ & $94 \pm 45$ & $83 \pm 34$ & $95 \pm 21$ & $120 \pm 28$ & $90 \pm 41$ \\
\hline LV1 (ml.kg-1.- $\left.\mathrm{min}^{-1}\right)$ & $12.5 \pm 3,8$ & $15.6 \pm 4,2$ & $11.2 \pm 4,2$ & $11.2 \pm 3,9$ & $13.3 \pm 2,3$ & $14.2 \pm 2,2$ \\
\hline HR LV1 (bpm) & $99 \pm 13$ & $103 \pm 20$ & $95 \pm 22$ & $98 \pm 21$ & $98 \pm 16$ & $94 \pm 14$ \\
\hline LV2 (ml.kg-1. $\left.\mathrm{min}^{-1}\right)$ & $16.7 \pm 4,8$ & $18.0 \pm 4,5$ & $14.5 \pm 6,1$ & $15.0 \pm 5,0$ & $15.9 \pm 2,3$ & $17.1 \pm 2,5$ \\
\hline HR LV2 (bpm) & $107 \pm 16$ & $113 \pm 22$ & $106 \pm 20$ & $108 \pm 22$ & $108 \pm 15$ & $112 \pm 16$ \\
\hline
\end{tabular}

patients showed a lesser reach in a shorter period than that which the literature characterized as an indication of poor prognosis. ${ }^{16}$ The fact that no changes in the OUES were observed after the physical training may well be related to the characteristics of our sample, which presents an earlier beginning of lactic acidosis during the hyperventilation test, observed by the ventilatory threshold, determined by the central and peripheral incapacity during physical exercise. ${ }^{28}$

Other important records analyzed in this study concern the prognostic implications of the $\mathrm{VO}_{2}$ and $\mathrm{HR}$ recovery values after ending the cardiopulmonary exercise test. No changes in these indicators were observed, which is in accordance with findings from Myers et al., ${ }^{29}$ However, Myers did observe an increase in the $\mathrm{VO}_{2}$ peak in response to eight weeks of aerobic training (walking and cycling) in CHF patients. It is impossible to determine a specific cause for these results. Nevertheless, it can be speculated that the delay in $\mathrm{VO}_{2}$ recovery is related to the difficulty to recover energy stored in the main muscles used in the cardiopulmonary exercise test. ${ }^{30}$
The HR recovery kinetics, as a prognostic marker, is well-established and is used as an independent factor of mortality, even in CHF patients that take $\beta$-blockers. ${ }^{31,32}$ Therefore, the results of this study showed that the reduction was greater than $12 \mathrm{bpm}$, in the first minute after ending the exercise. This value was used as a cutoff point to define the mortality in patients with left ventricular dysfunction, ${ }^{33}$ whose cut-off point was $\leq 16$ $\mathrm{bpm}$, in an active recovery protocol. Only in the CON group was a reduction of $\leq 16 \mathrm{bpm}$ observed at the end of the study. The fall in HR immediately after physical exercise occurs in response to the autonomous control, more precisely, the activation of the parasympathetic nerve system due to the sympathetic nerve activity. This adjustment is also an important prognostic marker and is associated with a reduction in the risk of death. ${ }^{14}$

In the evaluation of other variables of the cardiopulmonary exercise test, the time to reach the $\mathrm{VO}_{2}$ peak also reflects the capacity to predict mortality in patients with CHF and a reduced LVEF. ${ }^{34}$ This study showed that the time of duration in the HIIT group was 
greater for the $\mathrm{VO}_{2}$ peak, LV1, and LV2, which is directly related to the positive adaptations of the training. In another study, the authors present results that run in line with those from our study. ${ }^{35}$

Our results demonstrate consistent improvements in the parameters related to one's tolerance to physical exercise, which is a key characteristic in CHF patients. Appropriately, our study presents important clinical implications for the state of health concerning the autonomy and performance of one's DLAs. On the other hand, our study presents a series of limitations that should be observed, one of which refers to the $\mathrm{VO}_{2}$ evaluation protocol of $\mathrm{CHF}$ patients. The reach of $\mathrm{VO}_{2}$ peak does not translate into the maximum capacity of exercise and gas exchanges referent to the evaluation and prescription of adequate intensities for physical training. Thus, the $\mathrm{VO}_{2}$ max would be more accurate, but with greater risk and discomfort for the participants. Another limitation is the relatively small sample. This makes it impossible to stratify the results, such as severity of $\mathrm{CHF}$, time of diagnosis, and inclusion in the rehabilitation program, among others.

\section{Conclusions}

This retrospective study showed that both the conventional protocol (CAT) and a high-intensity interval training (HIIT), carried out over a 10-week period, promoted positive adaptations in the capacity of physical exercise, with an increase in time, speed, and treadmill slopes to achieve important $\mathrm{CHF}$ prognostic markers. However, the HIIT group showed improvement in a greater number of variables. Based on these results, we support the concept of the need to measure submaximal variables that reflect a better integration between the mechanisms related to changes in the cardiovascular, pulmonary, and musculoskeletal system of these patients, in attempt to reintegrate $\mathrm{CHF}$ patients into social life within society.

\section{Author Contributions}

Conception and design of the research: Busin D, Lehnen AM, Tairova OS, Lago PD, Nunes RB. Acquisition of data: Busin D, Tairova OS, Comparsi EP, Carneiro D, Potter M. Analysis and interpretation of the data: Busin D, Lehnen AM, Tairova OS, Comparsi EP, Carneiro D, Potter M, Deresz LF, Lago PD, Nunes RB. Statistical analysis: Busin D, Lehnen AM, Tairova OS, Comparsi EP, Carneiro D, Potter M, Deresz LF, Lago PD, Nunes RB. Obtaining financing: Tairova OS. Writing of the manuscript: Busin D, Lehnen AM, Tairova OS, Comparsi EP, Deresz LF, Lago PD, Nunes RB. Critical revision of the manuscript for intellectual content: Busin D, Lehnen AM, Tairova OS, Comparsi EP, Carneiro D, Potter M, Deresz LF, Lago PD, Nunes RB.

\section{Potential Conflict of Interest}

No potential conflict of interest relevant to this article was reported.

\section{Sources of Funding}

There were no external funding sources for this study.

\section{Study Association}

This article is part of the thesis of master submitted by Diego Busin, from Universidade Federal de Ciências da Saúde de Porto Alegre.

\section{Ethics Approval and Consent to Participate}

This study was approved by the Ethics Committee of the Universidade Federal de Ciências da Saúde de Porto Alegre under the protocol number 3.055.126. All the procedures in this study were in accordance with the 1975 Helsinki Declaration, updated in 2013. Informed consent was obtained from all participants included in the study. 


\section{References}

1. Mosterd A, Hoes AW. Clinical epidemiology of heart failure. Heart 2007; 93(9): 1137-46.

2. Writing Group M, Mozaffarian D, Benjamin EJ, Go AS, Arnett DK, Blaha MJ et al. Heart Disease and Stroke Statistics-2016 Update: A Report From the American Heart Association. Circulation 2016; 133(4): e38-360.

3. Leon AS, Franklin BA, Costa F, Balady GJ, Berra KA, Stewart KJ et al. Cardiac rehabilitation and secondary prevention of coronary heart disease: an American Heart Association scientific statement from the Council on Clinical Cardiology (Subcommittee on Exercise, Cardiac Rehabilitation, and Prevention) and the Council on Nutrition, Physical Activity, and Metabolism (Subcommittee on Physical Activity), in collaboration with the American association of Cardiovascular and Pulmonary Rehabilitation. Circulation 2005; 111(3): 369-76.

4. Belardinelli R, Georgiou D, Cianci G, Purcaro A. 10-year exercise training in chronic heart failure: a randomized controlled trial. J Am Coll Cardiol 2012; 60(16): 1521-8.

5. Nilsson BB, Westheim A, Risberg MA. Effects of group-based highintensity aerobic interval training in patients with chronic heart failure. Am J Cardiol 2008; 102(10): 1361-5.

6. Pina IL, Apstein CS, Balady GJ, Belardinelli R, Chaitman BR, Duscha BD et al. Exercise and heart failure: A statement from the American Heart Association Committee on exercise, rehabilitation, and prevention. Circulation 2003; 107(8): 1210-25.

7. Piepoli M, Maugeri F, Campana M, Ferrari R, Giordano A, Scalvini S et al. Experience from controlled trials of physical training in chronic heart failure. Protocol and patient factors in effectiveness in the improvement in exercise tolerance. European Heart Failure Training Group. Eur Heart J 1998; 19(3): 466-75.

8. O'Connor CM, Whellan DJ, Lee KL, Keteyian SJ, Cooper LS, Ellis SJ et al. Efficacy and safety of exercise training in patients with chronic heart failure: HF-ACTION randomized controlled trial. JAMA 2009; 301(14): 1439-50.

9. Vanhees L, De Sutter J, Gelada SN, Doyle F, Prescott E, Cornelissen V et al. Importance of characteristics and modalities of physical activity and exercise in defining the benefits to cardiovascular health within the general population: recommendations from the EACPR (Part I). Eur J Prev Cardiol 2012; 19(4): 670-86.

10. Rognmo O, Hetland E, Helgerud J, Hoff J, Slordahl SA. High intensity aerobic interval exercise is superior to moderate intensity exercise for increasing aerobic capacity in patients with coronary artery disease. Eur J Cardiovasc Prev Rehabil 2004; 11(3): 216-22.

11. Tzanis G, Philippou A, Karatzanos E, Dimopoulos S, Kaldara E, Nana E et al. Effects of High-Intensity Interval Exercise Training on Skeletal Myopathy of Chronic Heart Failure. J Card Fail 2017; 23(1): 36-46.

12. Wisloff U, Stoylen A, Loennechen JP, Bruvold M, Rognmo O, Haram PM et al. Superior cardiovascular effect of aerobic interval training versus moderate continuous training in heart failure patients: a randomized study. Circulation 2007; 115(24): 3086-94.

13. Sue DY. Excess ventilation during exercise and prognosis in chronic heart failure. Am J Respir Crit Care Med 2011; 183(10): 1302-10.

14. Vicente-Campos D, Martin Lopez A, Nunez MJ, Lopez Chicharro J. Heart rate recovery normality data recorded in response to a maximal exercise test in physically active men. Eur J Appl Physiol 2014; 114(6): 1123-8.

15. Sun XG, Hansen JE, Garatachea N, Storer TW, Wasserman K. Ventilatory efficiency during exercise in healthy subjects. Am J Respir Crit Care Med 2002; 166(11): 1443-8

16. Hollenberg M, Tager IB. Oxygen uptake efficiency slope: an index of exercise performance and cardiopulmonary reserve requiring only submaximal exercise. J Am Coll Cardiol 2000; 36(1): 194-201.

17. Guazzi M, Adams V, Conraads V, Halle M, Mezzani A, Vanhees L et al. EACPR/AHA Scientific Statement. Clinical recommendations for cardiopulmonary exercise testing data assessment in specific patient populations. Circulation 2012; 126(18): 2261-74.
18. Cohen-Solal A, Laperche T, Morvan D, Geneves M, Caviezel B, Gourgon $R$. Prolonged kinetics of recovery of oxygen consumption after maximal graded exercise in patients with chronic heart failure. Analysis with gas exchange measurements and NMR spectroscopy. Circulation 1995; 91(12): 2924-32.

19. Pardaens K, Van Cleemput J, Vanhaecke J, Fagard RH. Peak oxygen uptake better predicts outcome than submaximal respiratory data in heart transplant candidates. Circulation 2000; 101(10): 1152-7.

20. Haykowsky MJ, Timmons MP, Kruger C, McNeely M, Taylor DA, Clark AM. Meta-analysis of aerobic interval training on exercise capacity and systolic function in patients with heart failure and reduced ejection fractions. Am J Cardiol 2013; 111(10): 1466-9.

21. Ulbricha A, Angartena V, Netto A, Sties S, Bündchen D, de Mara L et al Comparative effects of high intensity interval training versus moderate intensity continuous training on quality of life in patients with heart failure: Study protocol for a randomized controlled trial. 2016; 13: 21-8.

22. Kell RT, Bell G, Quinney A. Musculoskeletal fitness, health outcomes and quality of life. Sports Med 2001; 31(12): 863-73.

23. Cornelis J, Beckers P, Taeymans J, Vrints C, Vissers D. Comparing exercise training modalities in heart failure: A systematic review and meta-analysis. Int J Cardiol 2016; 221: 867-76.

24. Woods PR, Bailey KR, Wood CM, Johnson BD. Submaximal exercise gas exchange is an important prognostic tool to predict adverse outcomes in heart failure. Eur J Heart Fail 2011; 13(3): 303-10.

25. Davies LC, Wensel R, Georgiadou P, Cicoira M, Coats AJ, Piepoli MF et al. Enhanced prognostic value from cardiopulmonary exercise testing in chronic heart failure by non-linear analysis: oxygen uptake efficiency slope. Eur Heart J 2006; 27(6): 684-90.

26. Chua TP, Ponikowski P, Harrington D, Anker SD, Webb-Peploe K, Clark AL et al. Clinical correlates and prognostic significance of the ventilatory response to exercise in chronic heart failure. J Am Coll Cardiol 1997; 29(7): 1585-90.

27. Ferreira AM, Tabet JY, Frankenstein L, Metra M, Mendes M, Zugck $\mathrm{C}$ et al. Ventilatory efficiency and the selection of patients for heart transplantation. Circ Heart Fail 2010; 3(3): 378-86.

28. Baba R, Nagashima M, Goto M, Nagano Y, Yokota M, Tauchi N et al. Oxygen intake efficiency slope: a new index of cardiorespiratory functional reserve derived from the relationship between oxygen consumption and minute ventilation during incremental exercise. Nagoya J Med Sci 1996; 59(1-2): 55-62.

29. Myers J, Gianrossi R, Schwitter J, Wagner D, Dubach P. Effect of exercise training on postexercise oxygen uptake kinetics in patients with reduced ventricular function. Chest 2001; 120(4): 1206-11.

30. Sullivan MI, Higginbotham MB, Cobb FR. Increased exercise ventilation in patients with chronic heart failure: intact ventilatory control despite hemodynamic and pulmonary abnormalities. Circulation 1988; 77(3): 552-9.

31. Arena R, Myers J, Abella J, Peberdy MA, Bensimhon D, Chase P et al. The prognostic value of the heart rate response during exercise and recovery in patients with heart failure: influence of beta-blockade. Int J Cardiol 2010; 138(2): 166-73.

32. Dimopoulos S, Anastasiou-Nana M, Sakellariou D, Drakos S Kapsimalakou S, Maroulidis G et al. Effects of exercise rehabilitation program on heart rate recovery in patients with chronic heart failure. Eur J Cardiovasc Prev Rehabil 2006; 13(1): 67-73.

33. Cole CR, Blackstone EH, Pashkow FJ, Snader CE, Lauer MS. Heart-rate recovery immediately after exercise as a predictor of mortality. N Engl J Med 1999; 341(18): 1351-7.

34. Keteyian SJ, Patel M, Kraus WE, Brawner CA, McConnell TR, Pina IL et al. Variables Measured During Cardiopulmonary Exercise Testing as Predictors of Mortality in Chronic Systolic Heart Failure. J Am Coll Cardiol 2016; 67(7): 780-9.

35. Brawner CA, Shafiq A, Aldred HA, Ehrman JK, Leifer ES, Selektor Y et al. Comprehensive analysis of cardiopulmonary exercise testing and mortality in patients with systolic heart failure: the Henry Ford Hospital cardiopulmonary exercise testing (FIT-CPX) project. J Card Fail 2015; 21(9): 710-8. 